\title{
Convex Optimization Algorithms for Multiple Source Localization Based on Received Signal Strength Measurements
}

\author{
Xiaodun Deng \\ Modern Education Technology Center, Xi'an International University, Xi'an, \\ China \\ dengxiaodun08@163.com
}

\begin{abstract}
Decaying with the increasing of signal propagation distance, Received Signal Strength (RSS) is used in the wireless localization due to its low cost and easily implementation. When the transmit power is unavailable, two convex optimization algorithms including semi definite programming (SDP), second order cone and semi definite programming $(S O C / S D P)$ are designed to estimate the source locations by relaxing the non-convex problem as convex optimization. The corresponding Cramér-Rao lower bound (CRLB) of the problem is derived. The simulations demonstrate that the SOC/SDP algorithm provides the similar accuracy performance compared with the SDP algorithm. However, the computational complexity of SOC/SDP is lower than that of the SDP due to the less variables and equality constraints. When perfect knowledge of the path loss exponent is available, the simulations also show that the accuracy performance of the proposed convex optimization algorithms degrades as the path loss exponent increases.
\end{abstract}

Keywords: wireless sensor networks; localization; received signal strength; convex optimization

\section{Introduction}

Localization techniques play a critical role in most of wireless sensor network (WSN) applications such as coverage calculation, event detection, object tracking, and geometric routing [1-3]. In such applications, sensor nodes are categorized into anchor nodes and source nodes. The main difference between them is that the anchor nodes know their locations, for instance with the help of GPS, whereas the locations of source nodes are unknown and required to be estimated.

To locate the source nodes with these anchor nodes, ranging information is required to be measured based on one or more physical parameters of the radio signal exchanged between the anchor nodes and the sensor nodes which are in communication range of each other. These measurements include the time of arrival (TOA) [4, 5], time difference of arrival (TDOA) [6] and received signal strength (RSS) [7, 8]. There is a tradeoff in the techniques, which rely on these parameters, in terms of implementation complexity and localization accuracy. Among these techniques, TOA-based range estimates are inherently more accurate than the alternatives, while the RSS-based techniques provide the solutions with low-cost and easily implement [9].

By using one or several aforementioned parameters, the localization problem can be modeled as an estimation problem. When the statistics of the measurement error are known, the maximum likelihood (ML) estimator is asymptotically optimal. However, the numerical solution of ML estimation highly depends on the initial point provided for the iterative solver. A poor initialization often leads to a very bad estimation. The nonconvex ML estimator would be possible to trap in a local minimum. To overcome shortcoming of the ML estimator, several researchers have proposed the linear estimator. By converting the nonlinear equations into a set of linear equations with respect to the 
locations of the source nodes, the linear estimator [10] provides closed-form solutions for the source location estimation. However, the accuracy performance is not very well at larger noise.

Another method is the use of the convex optimization technique by relaxing the nonconvex and nonlinear problem as convex optimization including the semi definite programming (SDP) [11, 12], second order cone programming (SOCP) [13] and their mixture. By taking advantage of the convex optimization technique, the global minimum of a convex problem can be quickly and efficiently found. The SDP estimators in [11] and [12] are proposed by exploiting the pairwise distance information. SDP solution is an approximate ML estimate due to the relaxation of optimization equations, but it has a complicated structure and high computational complexity. Compared with the SDP algorithm, the SOCP runs faster than the SDP algorithm due to the less variables and equality constraints as for the same optimization model.

When the anchor nodes transmit the signal received by the source nodes, the RSS measurements of the source nodes depend on the transmit power of the anchor nodes. However, most researches of RSS-based wireless localization assume that the transmit power is known. In fact, the transmit power will be subject to a large fluctuation because its value is dependent on the height and orientation of the node antenna, as well as antenna gain and its battery which will decrease with time. In [14] linear least squares is utilized to determine the location of the source node when path loss model parameters are unknown. The performance shows that the presented method outperforms other off-the-shelf source node localization algorithms when path-loss model parameters are unknown. The optimal power allocation policy is first derived in [15] for the case that the anchor nodes estimate their own locations with no error. The results show that a substantial reduction in power consumption can be achieved by optimal allocation of the transmission power.

In this paper two convex optimization algorithms including SDP and SOC/SDP are proposed to estimate the source locations when the transmit power of the anchor nodes is unavailable. Here the RSS measurement is assumed to be the log-normal shadowing. The path loss exponent and shadow fading variances in the model are known a priori through a calibration phase. By relaxing the non-convex target function as convex optimization, the SDP and SOC/SDP algorithms provide robust solutions to the source location estimation.

This paper mainly presents convex optimization algorithms including SDP and SOC/SDP for RSS-based wireless localization when the transmit power is unavailable. The rest of this paper is structured as follows. Section 2 presents the problem specification of localization model in the network. Section 3 in detail describes the localization design with convex optimization algorithms. Section 4 derives the corresponding performance of CRLB. Section 5 analyzes the simulation results. The conclusion is represented in Section 6. This paper contains a number of symbols. Following the convention, we represent the matrices as bold case letters. If we denote the matrix as $(*),(*)^{-1}$ represents matrix inverse. $[\mathbf{A}]_{i, j}$ denotes the element at the $i$ th row and $j$ th column of matrix $\mathbf{A}$. For arbitrary symmetric matrices $\mathbf{A}, \mathbf{A} \succeq 0$ means that $\mathbf{A}$ is positive semi definite.

\section{Localization Model}

Consider a wireless network with $N$ anchor nodes of known locations denoted by vectors $\mathbf{a}_{1}, \cdots, \mathbf{a}_{M}$. The problem is to localize $M$ unknown indistinguishable source nodes whose locations are denoted by vectors $\mathbf{x}_{1}, \cdots, \mathbf{x}_{N}$. In the proposed model, the anchor nodes transmit the wireless signal which is received by the source nodes. Considering a statistical path loss model, the $\mathrm{dBm}$ value of the average 
power collected from the $i$ th source node and corresponding to the signal transmitted by the $j$ th anchor node is given by

$$
P_{i, j}=P_{j, 0}-10 \beta \log _{10} d_{i, j}+n_{i, j}
$$

where $P_{j, 0}$ is the power transmitted by the $j$ th anchor node and received by the $i$ th source node at the reference distance of $1 \mathrm{~m} . \beta$ and $d_{i, j}$ is the path loss exponent and the distance for the link between the $i$ th source node and the $j$ th anchor node. $n_{i, j}$ is the corresponding shadowing term modeled as independent zero-mean Gaussian random variables with standard deviation $\delta_{i, j}^{2}$.

In this case, both the source coordinates and the transmit powers of the anchor nodes are unknown and should be estimated. Denote the positions of the source nodes and transmit powers of all anchor nodes in vector form by

$$
\begin{gathered}
\mathbf{x}=\left[\begin{array}{llll}
\mathbf{x}_{1}^{T} & \mathbf{x}_{2}^{T} & \cdots & \mathbf{x}_{N}^{T}
\end{array}\right]^{T} \\
\mathbf{p}=\left[\begin{array}{llll}
P_{1,0} & P_{2,0} & \cdots & P_{M, 0}
\end{array}\right]
\end{gathered}
$$

Our aim is to estimate $\mathbf{x}$ based on the received signal strength measurements where the transmit power vector $\mathbf{p}$ is unknown.

To unique positioning the source node, the requisite number of RSS measurements is now discussed. In the two-dimensional space, it is well known that at least three non collinear transmitters and measurements are used to uniquely locate a source node. Hence we need at least $3 N$ distance measurements to locate $N$ nodes assuming that the transmit powers are known.

When $\mathbf{p}_{0}$ is unknown, the requisite number of RSS measurements is increased to $3 N+M$ due to the extra $M$ unknown parameters. Since there are at most $M N$ measurements in total, this leads to the inequality

$$
M N \geq 3 N+M
$$

Then we can obtain that

$$
M \geq \frac{3 N}{N-1}, \quad N \geq 2
$$

When $N=2, M$ must be meet that $M \geq 6$. (4) Also means that

$$
N \geq \frac{M}{M-3}, \quad M \geq 4
$$

Where $M=4, N$ must be meet that $N \geq 4$.

The well-known maximum likelihood (ML) estimation for the proposed model can be represented as the following optimization problem

$$
\min _{\mathbf{x}, \mathbf{p}} \sum_{i=1}^{N} \sum_{j=1}^{M} \frac{1}{\delta_{i, j}^{2}}\left(P_{i, j}-P_{j, 0}+10 \beta \log _{10} d_{i, j}\right)^{2}
$$

However, the numerical solution of the ML estimation depends on the initial conditions or may suffer from local minima and even divergence problems. The semi definite programming algorithm converts the non-convex formulation of (7) to a convex optimization program which always guarantees a global solution. Another method to avoid the shortcoming of the ML estimation is the linear estimator. The linear estimator 
transforms the nonlinear equations to a set of linear ones and obtains the closed-form solution to the source position. In the following, we in detail present the designed semi definite programming algorithm for the proposed RSS-based localization model when the transmit power is unavailable.

\section{Semidefinite Programming Algorithm}

To obtain the linear expression, (1) is rewritten as

$$
10 \beta \log _{10} d_{i, j}=P_{j, 0}-P_{i, j}+n_{i, j}
$$

By dividing both sides of $(8)$ by $5 \beta,(8)$ is further written as

$$
\log _{10} d_{i, j}^{2}=\frac{P_{j, 0}-P_{i, j}+n_{i, j}}{5 \beta}
$$

Taking the power of 10 on both sides of (9) yields

$$
d_{i, j}^{2}=10^{\frac{P_{j, 0}-P_{i, j}+n_{i, j}}{5 \beta}}
$$

For sufficiently small noise, the right hand side of (10) can be approximated using the first-order Taylor series expansion as

$$
d_{i, j}^{2}=\mu_{i, j} \rho_{j}+\varepsilon_{i, j}
$$

where $\mu_{i, j}=10^{\frac{-P_{i, j}}{5 \beta}}, \rho_{j}=10^{\frac{P_{j, 0}}{5 \beta}}, \lambda_{i, j}=\mu_{i, j} \rho_{j}, \quad \varepsilon_{i, j}=\frac{\lambda_{i, j} \ln 10}{5 \beta} n_{i, j}$ is the noise modeled as a zero-mean Gaussian random variable with variance $v_{i, j}^{2}$.

$$
v_{i, j}^{2}=\frac{\lambda_{i, j}^{2}(\ln 10)^{2}}{25 \beta^{2}} \delta_{i, j}^{2}
$$

So the ML estimation can be reformulated as

$$
\min _{\mathbf{x}, \boldsymbol{\rho}} \sum_{i=1}^{N} \sum_{j=1}^{M} \frac{1}{v_{i, j}^{2}}\left(d_{i, j}^{2}-\mu_{i, j} \rho_{j}\right)^{2}
$$

where $d_{i, j}^{2}=\left\|\mathbf{x}_{i}-\mathbf{a}_{j}\right\|^{2}, \boldsymbol{\rho}=\left[\rho_{j} \mid j=1,2, \cdots, M\right]$ denotes the unknown parameter vector corresponding to the transmit power. The unknown parameter vector along with the source locations is estimated by using the ML estimation. Producing a new variable

$$
\mathbf{Z}=\left[\begin{array}{cc}
\mathbf{I}_{2} & \mathbf{x} \\
\mathbf{x}^{T} & \mathbf{x}^{T} \mathbf{x}
\end{array}\right]
$$

Then the parameter $d_{i, j}^{2}$ can be further rewritten as

$$
d_{i, j}^{2}=\left\lceil\begin{array}{c}
\mathbf{a}_{j} \\
-\mathbf{e}_{i}
\end{array}\right]^{T} \mathbf{Z}\left[\begin{array}{c}
\mathbf{a}_{j} \\
-\mathbf{e}_{i}
\end{array}\right]
$$

where $\mathbf{e}_{i}$ is an $N \times 1$ column vector with 1 at the $i$ th entry and 0 's elsewhere. By relaxing $\mathbf{Z} \succeq \mathbf{0}_{N+2}$, we construct a semidefinite relaxation form of (13) which can be 
rewritten as

$$
\begin{gathered}
\min _{\mathbf{Z}, \boldsymbol{\rho}} \sum_{i=1}^{N} \sum_{j=1}^{M} \frac{1}{v_{i, j}^{2}}\left(d_{i, j}^{2}-\mu_{i, j} \rho_{j}\right)^{2} \\
\text { s.t. } \quad d_{i, j}^{2}=\left[\begin{array}{c}
\mathbf{a}_{j} \\
-\mathbf{e}_{i}
\end{array}\right]^{T} \mathbf{Z}\left[\begin{array}{c}
\mathbf{a}_{j} \\
-\mathbf{e}_{i}
\end{array}\right] \\
\mathbf{Z} \succeq \mathbf{0}_{N+2}
\end{gathered}
$$

For avoiding the local minima, (13) is converted into the SDP optimization problem

$$
\begin{array}{ll}
\min _{\mathbf{Z}, \boldsymbol{\rho}, \boldsymbol{\alpha}} & \sum_{i=1}^{N} \sum_{j=1}^{M} \alpha_{i, j} \\
\text { s.t. } & {\left[\begin{array}{c}
\alpha_{i, j} \\
\frac{1}{v_{i, j}}\left(d_{i, j}^{2}-\mu_{i, j} \rho_{j}\right) \\
d_{i, j}^{2}=\left[\begin{array}{c}
\mathbf{a}_{j} \\
-\mathbf{e}_{i}
\end{array}\right]^{T} \mathbf{Z}\left[\begin{array}{c}
\mathbf{a}_{j} \\
-\mathbf{e}_{i}
\end{array}\right] \\
\mathbf{Z} \succeq \mathbf{0}_{N+2}
\end{array}\right]}
\end{array}
$$

where $\boldsymbol{\alpha}=\left[\alpha_{i, j} \mid i=1,2, \cdots, N, j=1,2, \cdots, M\right]$. (17) provides an SDP solution to the proposed RSS localization model. But the SDP algorithm runs slower due to a larger number of the variables and the equality constraints. So an alternative algorithm is the SOCP algorithm which has less variables and equality constraints. (17) is equivalent to the following optimization problem

$$
\begin{aligned}
& \min _{\mathbf{Z}, \mathbf{p}, \mathbf{a}, \mathbf{u}, v} v \\
& \text { s.t. }\|\mathbf{u}\| \leq v \\
& \frac{1}{v_{i, j}}\left(d_{i, j}^{2}-\mu_{i, j} \rho_{j}\right)=u_{i, j} \\
& d_{i, j}^{2}=\left[\begin{array}{c}
\mathbf{a}_{j} \\
-\mathbf{e}_{i}
\end{array}\right]^{T} \mathbf{Z}\left[\begin{array}{c}
\mathbf{a}_{j} \\
-\mathbf{e}_{i}
\end{array}\right] \\
& \quad \mathbf{Z} \succeq \mathbf{0}_{N+2}
\end{aligned}
$$

where $\mathbf{u}=\left[u_{i, j} \mid i=1,2, \cdots, N, j=1,2, \cdots, M\right]$. The solutions to (17) and (18) are determined by the transmit power which is not available. Preliminarily considering $\lambda_{i, j}$ as one we obtain the initial estimate $\lambda_{i, j}$. Then putting the initial estimate into these optimization expressions would produce a better solution for the positions of source nodes.

\section{CRLB Performance}

The CRLB matrix provides a lower bound on the covariance of any unbiased location estimator and is equal to inverse of fisher information matrix (FIM). In this section, the position CRLB of source node with unknown transmit power is derived. Since the transmit power is not available to the estimator, it should also be taken into account as an unknown parameter. Let $\boldsymbol{\varphi}=\left[\begin{array}{ll}\mathbf{x}^{T} & \mathbf{p}_{0}^{T}\end{array}\right]^{T}$. The FIM is denoted as $\mathbf{F}$, which is obtain by 


$$
\mathbf{F}=-\frac{\partial^{2} \ln P(\mathbf{p} \mid \varphi)}{\partial \varphi^{2}}
$$

where $P(\mathbf{p} \mid \boldsymbol{\varphi})=\prod_{i=1}^{N} \prod_{j=1}^{M} \frac{1}{\sqrt{2 \pi} \delta_{i, j}} \exp \left\{-\frac{\left(p_{i, j}-p_{j, 0}+10 \log _{10} d_{i, j}\right)^{2}}{2 \delta_{i, j}^{2}}\right\}, \quad \mathbf{F} \in \mathrm{R}^{(2 N+M) \times(2 N+M)}$.

Therefore, the elements of matrix $\mathbf{F}$ can be further represented as

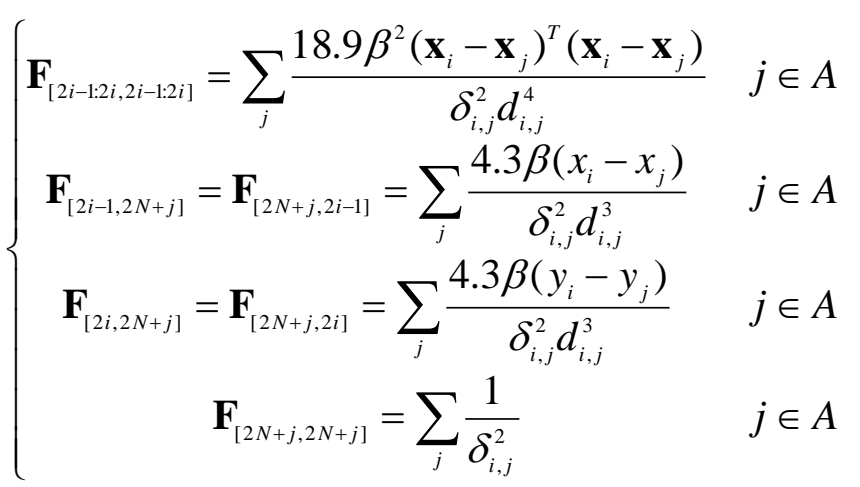

where $j \in A$ represents the source node $i$ can communicate with anchor node $j$. The CRLB of the unknown parameters is the diagonal element of the inverse of the FIM. So the CRLB of source node position is written as

$$
\operatorname{CRLB}\left(\mathbf{x}_{[r]}\right)=\mathbf{F}_{[r, r]}^{-1}
$$

where $r=1,2, \cdots, 2 N$. Given the FIM the position CRLB of source node $i$ is obtained by

$$
\operatorname{CRLB}\left(\mathbf{x}_{i}\right)=\mathbf{F}_{[2 i-1,2 i-1]}^{-1}+\mathbf{F}_{[2 i, 2 i]}^{-1}
$$

\section{Evaluation}

When the transmit power of the anchor node is assumed to be unknown, the SDP and SOC/SDP algorithms are proposed for RSS-based sensor localization. The proposed algorithms do not rely on the initialization and ensure the global convergence. Computer simulations were conducted to evaluate the robust performance of the proposed SDP and SOC/SDP algorithms. The proposed SDP and SOC/SDP are all implemented by the CVX toolbox using SeDuMi as the solver.

\subsection{Impact of the Shadow Fading}

Considering the configuration of the network given in Fig. 1, we perform Monte Carlo simulations with 500 ensemble runs to evaluate the root mean square error (RMSE) of the location estimation. To test the performance of proposed algorithms, we conduct a group of simulations with 15 nodes deployed in a $20 \mathrm{~m} \times 20 \mathrm{~m}$ square region. 5 anchor nodes are set at $(0,0),(0,20),(20,0),(20,20)$ and $(10,10)$ and used to locate other 10 source nodes deployed in the region randomly. We assume that all anchor-source connections are measurable. All transmit power $p_{j, 0}(j=1,2, \cdots, 5)$ of the anchor nodes are set to $-45 \mathrm{~dB}$ and assumed to be unknown. The shadow fadings $n_{i, j}$ are zero-mean white Gaussian variables with known identical variances of $\delta_{i, j}^{2}=\delta^{2}$. Figure 1 plots the geometry and connectivity of deployed 15 sensor nodes. 


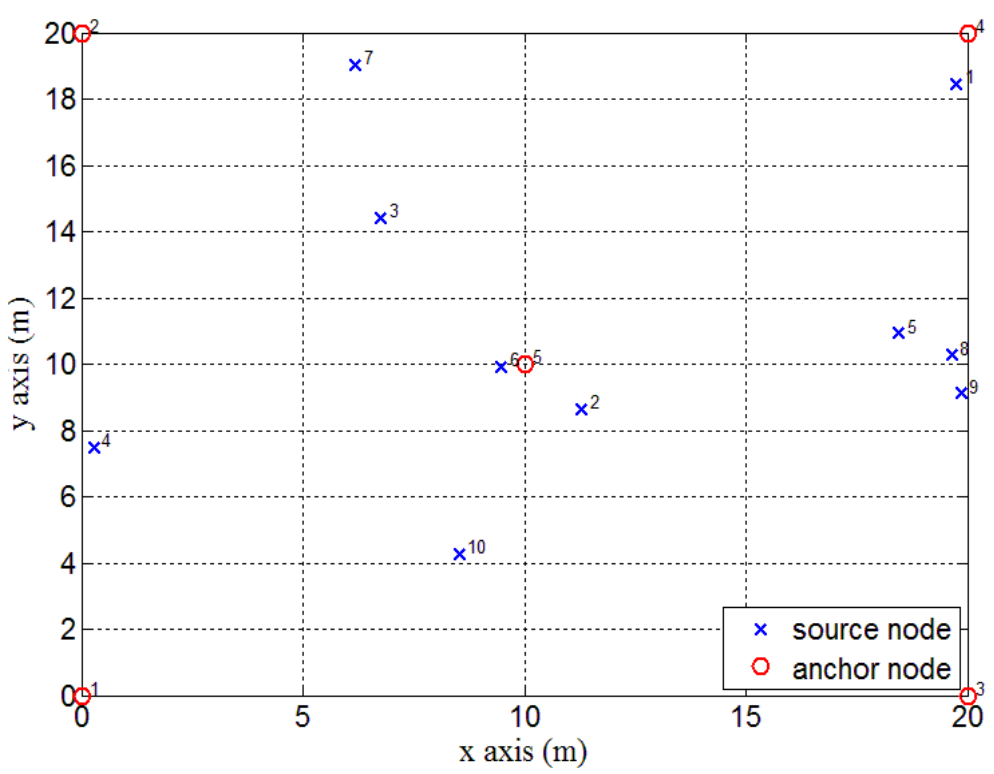

Figure 1. Geometry of Deployed 15 Sensor Nodes

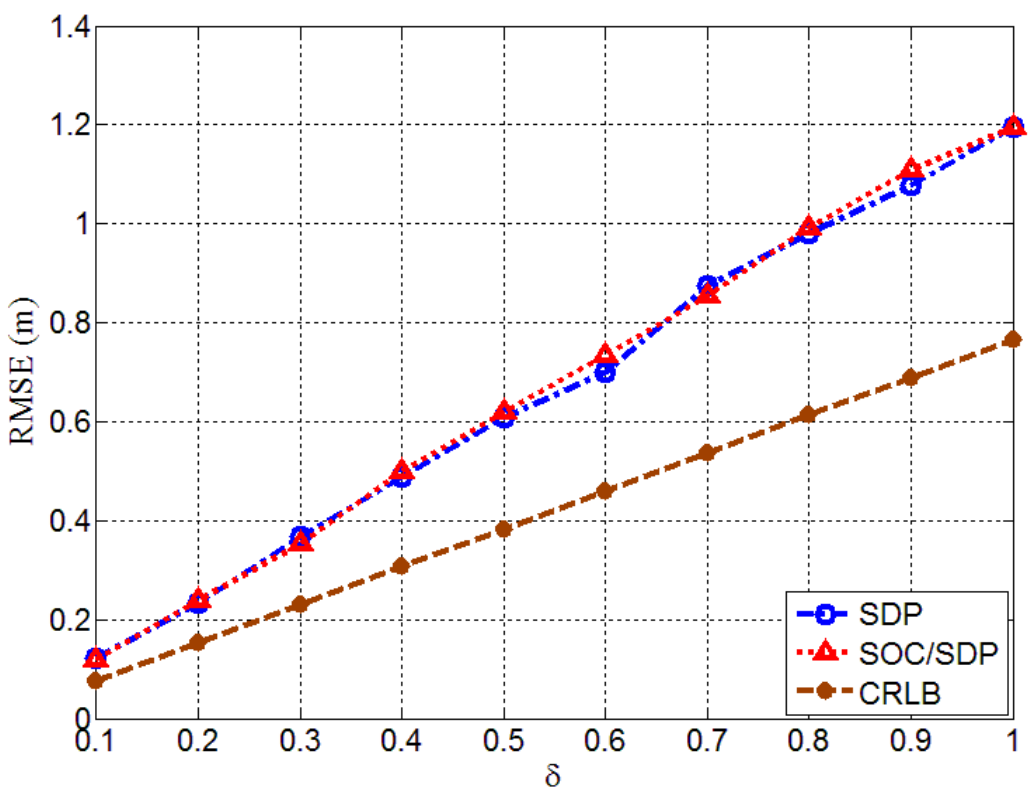

Figure 2. Impacts of Shadow Fading

Considering the configuration of the network given in Figure 3, we perform Monte Carlo simulations with 500 ensemble runs to evaluate the root mean square error (RMSE) of the location estimation. When the variances $\delta^{2}$ of shadow fading are varied from $0.1^{2}$ to $1^{2}$ and PLE value $\beta$ is set at 4 , the average RMSE of 10 source nodes versus shadow fading is plotted in Figure 2.

It can be seen from Figure 2 that the RMSE performance of SOC/SDP is very similar with the mixed SOC/SDP algorithm. The RMSE performance degrades as the shadow fading increases. When the shadow fading is set at 0.1 , the average RMSEs are also equal to $0.12 \mathrm{~m}$ with SDP and SOC/SDP algorithms. When the shadow fading is increased to 1, the average RMSEs are also increased to $1.2 \mathrm{~m}$ with SDP and SOC/SDP algorithms. The position RMSE is approximately linear with the shadow fading. Compared the optimal CRLB performance, the solutions of SDP and SOC/SDP algorithms are not very well due to the convex optimization 
relaxation.

\subsection{CPU Runtime}

The SOC/SDP algorithm provides the similar performance with the SDP algorithm. However, the SDP algorithm runs slower than the SDP algorithm due to the larger number of variables and equality constraints. In Table 1, we compare the average CPU runtime of the proposed algorithms. The running time is measured by averaging over 500 noise realizations when the standard deviation of the shadow fading is set to $4 \mathrm{~dB}$. When the network configuration is set as Figure 1. The number of variables and equality constraints are 349 and 103 for the SDP algorithm, respectively. However, the number of variables and equality constraints are reduced to 200 and 53 for the SOC/SDP algorithm. The average running time of SDP algorithm is $295 \mathrm{~ms}$. As for the same network configuration, the average running time of SOC/SDP algorithm is $201 \mathrm{~ms}$.

Table 1. CPU Runtime Comparison of Different Algorithms for the Network in Figure 1

\begin{tabular}{|c|c|c|c|c|}
\hline Algorithm & Measurements & Variables & Equality constraints & CPU runtime \\
\hline SDP & 50 & 349 & 103 & $295 \mathrm{~ms}$ \\
\hline SOC/SDP & 50 & 200 & 53 & $201 \mathrm{~ms}$ \\
\hline
\end{tabular}

\subsection{RMSE Performance of Different Source Node}

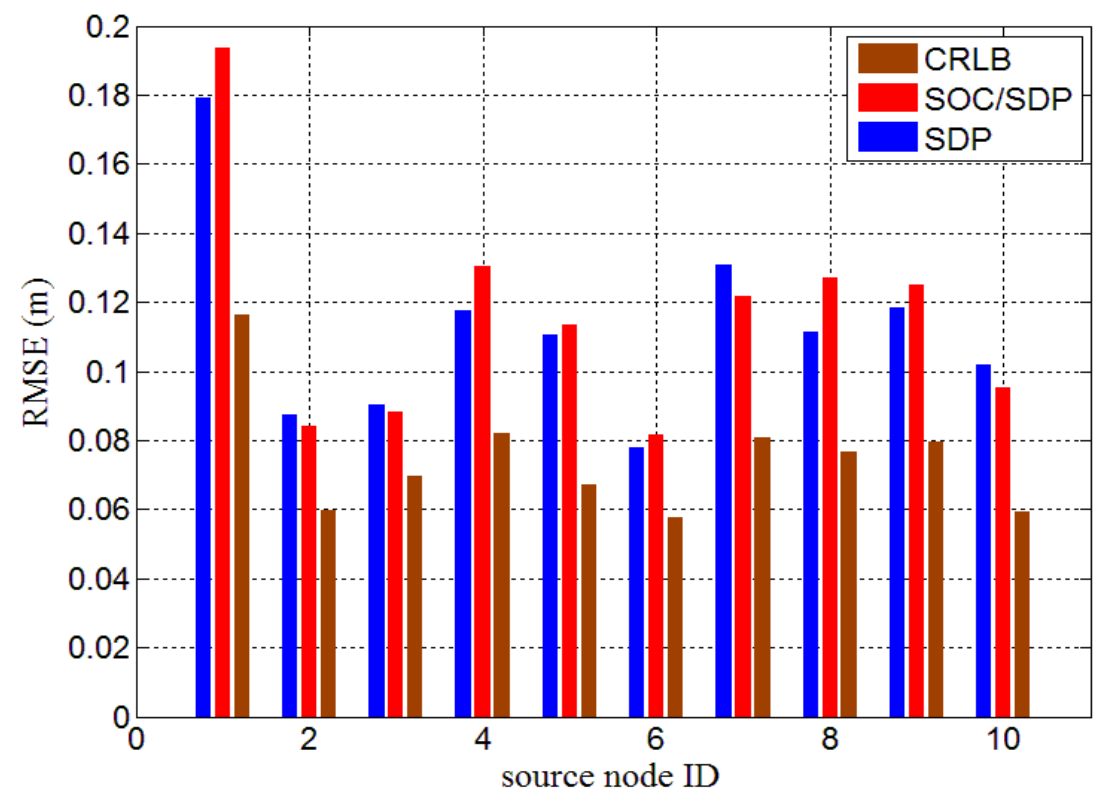

Figure 3. Performance Comparison of Different Source Node

Specific to the position of a source node, the positions of anchor nodes affect the positioning accuracy greatly. When the shadow fading is set to and PLE value $\beta$ is set at 4, the RMSE comparison of 10 source node is plotted in Figure 3 . Since located by different anchor nodes, the RMSE of each located node is diverse. The RMSE of source node ID 1 is $0.180 \mathrm{~m}$ in SDP algorithm and $0.193 \mathrm{~m}$ in SOC/SDP algorithm. However, the RMSE of source node ID 2 is only $0.087 \mathrm{~m}$ in SDP algorithm and $0.084 \mathrm{~m}$ in SOC/SDP algorithm. It also can be seen that the SOC/SDP algorithm provides the similar performance with the SDP algorithm. 


\subsection{Path Loss Exponent}

The estimate accuracy of the source node location highly relies on the path loss exponent value by using the RSS measurements. Generally, in wireless localization, the path loss exponent related with the environment is obtained through experimental analysis. We also investigate the effect of path loss exponent knowledge on the performance of the proposed algorithms. Similarly, when the network configuration is setup as Figure 1, the average RMSE of all source nodes is selected to evaluate the impacts of path loss exponent. When shadow fading is set at 0.1 and the path loss exponent $\beta$ is varied from 2 to 6 , the RMSE performance is plotted in Figure 4. The RMSE is decreased as path loss exponent $\beta$ increases. When $\beta$ is set at 2 , the RMSE of the proposed SDP and SOC/SDP algorithms is $0.23 \mathrm{~m}$. However it is reduced to $0.08 \mathrm{~m}$ when the path loss exponent $\beta$ is set at 6 . The simulations indicate that, for different path loss exponent parameters, the proposed SOC/SDP provides the similar localization accuracy with the SDP algorithm. However, the SOC/SDP runs faster that the SDP algorithm as for the same network configuration.

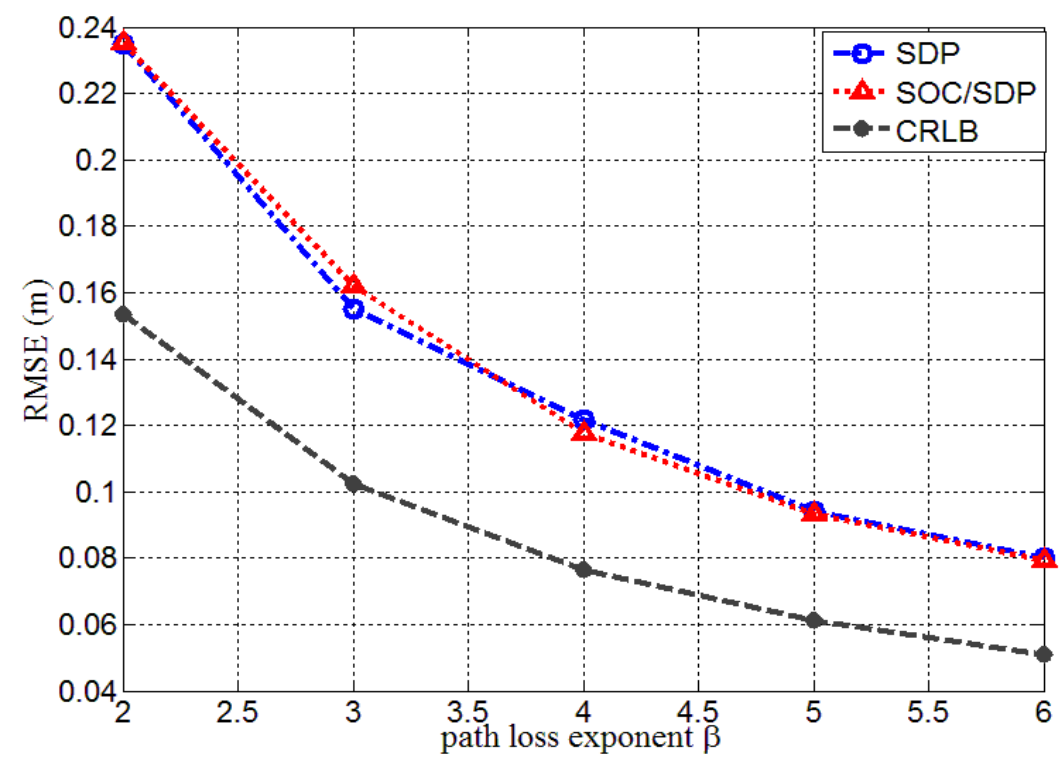

Figure 4. RMSE Performance versus PLE

\section{Conclusion}

When the transmit power is unavailable, the convex optimization algorithms are proposed to avoid the shortcoming of the ML estimation for the RSS-based sensor localization. By relaxing the RSS-based localization model to convex optimization problem, the SDP and SOC/SDP algorithms are designed. The localization accuracy degrades as the shadow fading increases. The accuracy performance becomes better as the path loss exponent increases. Compared with the SDP algorithm, the SOC/SDP algorithm provides the similar accuracy performance. However, the SOC/SDP runs faster than the SDP algorithm. Due to the convex relaxation, the proposed SDP and SOC/SDP algorithms cannot achieve the CRLB performance which provides the optimal accuracy. But the performance of the proposed SDP and SOC/SDP algorithms is robust since the algorithms do not rely on the initialization. 


\section{References}

[1] H. Sayed, A. Tarighat and N. Khajehnouri, "Network-based wireless location: Challenges faced in developing techniques for accurate wireless location information", IEEE Signal Processing Magazine, vol. 22, no. 4, (2005), pp. 24-40.

[2] M. Erol, H. Mouftah and S. Oktug, "Localization techniques for underwater acoustic sensor networks", IEEE Communication Magazine, vol. 48, no. 12, (2010), pp. 152-158.

[3] Y. Liu, Y. H. Hu and Q. Pan, "Distributed, robust acoustic source localization in a wireless sensor network", IEEE Trans. Signal Process., vol. 60, no. 8, (2012), pp. 4350-4359.

[4] I.Guvenc and C. C. Chong, "A survey on TOA based wireless localization and NLOS mitigation techniques", IEEE Communications Surveys \& Tutorials, vol. 11, no. 3, (2009), pp. 107-124.

[5] E. Xu, Z. Ding and S. Dasgupta, "Source localization in wireless sensor networks from signal time-ofarrival measurements", IEEE Transactions on Signal Processing, vol. 59, no. 6, (2011), pp. 2887-2897.

[6] L. Yang and K. C. Ho, "An approximately efficient TDOA localization algorithm in closed-form for locating multiple disjoint sources with erroneous sensor positions", IEEE Transactions on Signal Processing, vol. 57, no. 12, (2009), pp. 4598-4615.

[7] R. Vaghefi, M. Gholami, R. Buehrer and E. Strom, "Cooperative received signal strength-based sensor localization with unknown transmit powers", IEEE Transactions on Signal Processing, vol. 61, no. 6, (2013), pp. 1389-1403.

[8] J. Koo and H. Cha, "Localizing wifi access points using signal strength", IEEE Communication Letter, vol. 15, no. 2, (2011), pp. 187-189.

[9] X. Li, "Collaborative localization with received-signal strength in wireless sensor networks", IEEE Transactions on Vehicular Technology, vol. 56, no. 6, (2007), pp. 3807-3817.

[10] H. C. So and L. Lin, "Linear least squares approach for accurate received signal strength based source localization", IEEE Transactions on Signal Processing, vol. 59, no. 8, (2011), pp. 4035-4040.

[11] R. W. Ouyang, A. K. S. Wong, and C. T. Lea, "Received signal strength-based wireless localization via semidefinite programming: Noncooperative and cooperative schemes", IEEE Transactions on Vehicular Technology, vol. 59, no. 3, (2010), pp. 1307-1318.

[12] G. Wang and K. Yang, "A new approach to sensor node localization using RSS measurements in wireless sensor networks", IEEE Transaction Wireless Communications, vol. 10, no. 5, (2011), pp. 1389-1395.

G. N. Shirazi, M. B. Shenouda and L. Lampe, "Second Order Cone Programming for Sensor Network Localization with Anchor Position Uncertainty", IEEE Transactions on Wireless Communication, vol. 13, no. 2, pp. 949-963, 2014.

[13] Y. Xu, J. Zhou and P. Zhang, "RSS-Based Source Localization When Path-Loss Model Parameters are Unknown”, IEEE Communications Letters, vol. 18, no. 6, (2014), pp. 1055-1058.

[14] F. Yaghoubi and A. A. Abbasfar and B. Maham, "Energy-Efficient RSSI-Based Localization for Wireless Sensor Networks", IEEE Communications Letters, vol. 18, no. 6, (2014), pp. 973-976.

\section{Author}

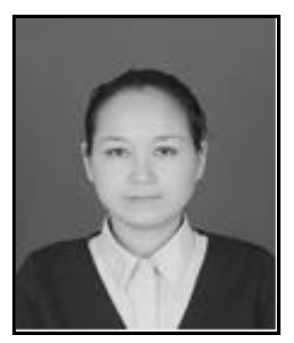

Xiaodun Deng, she is a lecturer at Modern Education Technology Center, Xi'an International University, main research areas are big data, computer network and artificial intelligence. 